\title{
Travelling waves in electroconvection of the nematic Phase 5: A test of the weak electrolyte model
}

\author{
Martin Treiber*, Nándor Éber**, Ágnes Buka**, and Lorenz Kramer* \\ * Universität Bayreuth, Theoretische Physik II, Universitätsstraße 30, D-95440 Bayreuth, \\ Germany \\ ** Research Institute for Solid State Physics of the Hungarian Academy of Sciences, H-1525 \\ Budapest, P.O.B.49, Hungary
}

(October 29, 2018)

We investigated travelling waves appearing as the primary pattern-forming instability in the nematic Phase 5 (Merck) in the planar geometry in order to test the recently developed weak electrolyte model of ac-driven electroconvection [M. Treiber and L. Kramer, Mol. Cryst. Liq. Cryst 261, 311 (1995)]. Travelling waves are observed over the full conductive range of applied frequencies for three cells of different layer thickness $d$. We also measured the elastic constants, the electric conductivity, and the dielectric constant. The other parameters of Phase 5 are known, apart from the (relatively unimportant) viscosity $\alpha_{1}$ and the two parameters of the weak electrolyte model that are proportional to the geometric mean of the mobilities, and the recombination rate, respectively. Assuming a sufficiently small recombination rate, the predicted dependence of the frequency of the travelling waves at onset (Hopf frequency) on $d$ and on the applied frequency agreed quantitatively with the experiments, essentially without fit parameters. The absolute value of the Hopf frequency implies that the geometric mean of the mobilities amounts to $1.1 \times 10^{-10} \mathrm{~m}^{2} /(\mathrm{Vs})$.

PACS 47.20-k, 47.65+a 


\section{INTRODUCTION}

Electrohydrodynamic convection (EHC) in nematic liquid crystals (NLC) is one of the most prominent phenomena employed for the study of pattern formation in anisotropic systems [1 3]. The system consists of a NLC with negative or only mildly positive dielectric anisotropy sandwiched between two glass plates with transparent electrodes. When applying an ac voltage with the circular frequency $\omega_{0}$ and increasing the rms $\bar{V}$ above a certain

threshold $\bar{V}_{c}\left(\omega_{0}\right)$, the non-convecting basic state becomes unstable to stationary or travelling rolls. In this work, we consider the usual planar-homogeneous geometry where the director of the NLC is aligned parallel to the plate electrodes.

The traditionally used hydrodynamic model of EHC originating from Helfrich [4 involves equations for the velocity field, the director, and the electric potential (or charge density). The NLC is considered as an anisotropic ohmic conductor. The origin of the conductivity is not specified. This standard hydrodynamic description [5] [7], including the three-dimensional formulation for EHC [8,3,9], is referred to as the Standard Model (SM). For a general introduction, see, e.g., the book of Blinov [10], or the reviews [1].

Many features in the conductive range of low ac frequencies are quantitatively described by the SM [3]. Examples are the threshold voltage as a function of the ac frequency $\omega_{0}$, the wave vector of the pattern, which describes the spacing and the angle of the rolls and includes the possibility of oblique rolls (where the roll axis is not perpendicular to the direction of planar alignment), [3,9] and, in the nonlinear regime, the amplitude of the roll pattern and secondary bifurcations leading in particular to (weak) defect turbulence [11 13]. Other features remain unexplained even on a qualitative level. Most notable are travelling waves (TW) which have been observed as early as 1978 [14. Later on, they were found by various groups in a broad parameter range in the NLCs MBBA [15,16], I52 [17, 18], and in Phase 5 [2,19]. TWs appear to be generic for relatively thin and clean cells and seem to be often favoured by higher external frequencies.

The recently developed weak electrolyte model (WEM) 20 predicts a Hopf bifurcation 
for these conditions, provided the recombination rate of mobile ions (see below) is sufficiently low. The WEM equations for the director and velocity fields are those of the SM, but the assumption of ohmic conduction is dropped and the conduction properties are explicitly modelled by two species of oppositely charged ionic charge carriers. The dynamics of the number densities $n^{+}(\boldsymbol{r}, t)$ and $n^{-}(\boldsymbol{r}, t)$ of the charge carriers is governed by drift relative to the fluid under an electric field and by a conventional dissociation-recombination reaction between neutral molecules and the ions. The WEM expresses the total space-charge density, which already appears in the SM, as the difference of the number densities of the two ionic species. In addition, the drift of the charges excites a new field, which can be expressed in terms of a variable local conductivity $\sigma_{\perp}(\boldsymbol{r}, t)$ perpendicular to the director ("charge-carrier mode").

The SM has two relevant time scales: the director-relaxation time $\tau_{d}=\gamma_{1} d^{2} /\left(K_{11} \pi^{2}\right)$ and the charge-relaxation time $\tau_{q}=\epsilon_{0} \epsilon_{\perp} / \sigma_{\perp}$. Here $\epsilon_{\perp}$ is the principal value of the dielectricconstant tensor perpendicular to the director, $\gamma_{1}$ is a rotational viscosity, and $K_{11}$ is the orientational elasticity for splay distortions. Typically, $\tau_{d}$ is $\mathcal{O}(1 \mathrm{~s})$, and $\tau_{q}$ is $\mathcal{O}\left(10^{-2} \mathrm{~s}\right)$. The WEM has two additional time scales [20,17]: a recombination time $\tau_{\text {rec }}$ for the relaxation towards the equilibrium of the dissociation-recombination reaction where $\sigma_{\perp}(\boldsymbol{r}, t)=\sigma_{\perp}^{\mathrm{eq}}$, and the transition time $\tau_{t}=d^{2} /(\bar{V} \mu)$ for a charge with the mobility $\mu=\mu_{\perp}^{+}+\mu_{\perp}^{-}$to traverse the cell under the critical applied voltage. The mobilities $\mu_{\perp}^{+}$and $\mu_{\perp}^{-}$are the principal values of the mobility tensors perpendicular to the director, see Eq. (3) below. The magnitude of $\tau_{t}$ will turn out to be of the order of $0.1 \mathrm{~s}$ whereas $\tau_{\text {rec }}>5 \mathrm{~s}$.

The $\mathrm{SM}$ is recovered in the limits $\tau_{t} / \tau_{q}>>1, \tau_{t} \omega_{0}>>1$, and $\frac{\tau_{t}}{\tau_{\mathrm{rec}}} \sqrt{\tau_{d} / \tau_{q}}>>1$. The ratio $\tau_{t} / \tau_{q}$ is a measure for the relative change of the charge-carrier densities induced by the space charges of the Carr-Helfrich mechanism. In our case the third inequality will be violated.

Assuming a recombination rate $\tau_{\text {rec }}^{-1}$ that is small compared to the circular Hopf frequency $\omega_{H}=2 \pi f_{H}$, the WEM predicts the Hopf frequency $f_{H}$ to be proportional to $\left(\mu_{\perp}^{+} \mu_{\perp}^{-}\right)^{1 / 2}\left(\sigma_{\perp}^{\mathrm{eq}}\right)^{-1 / 2} d^{-3}$, and depending otherwise only on $\omega_{0}$ and on the material param- 
eters of the SM. In experiments on the nematic I52 [17], the WEM explains succesfully the dependence of the Hopf frequency of the observed TWs on $\sigma_{\perp}^{\text {eq }}$ and on $\omega_{0}$. Measurements on a thicker cell seem also to confirm the predicted $d$ dependence, but no systematic measurements were made. Also, not all of the twelve material parameters of the SM are known for I52. Six parameters had to be fitted to the curves of the threshold voltage and of the roll angle as a function of $\omega_{0}$. These curves can be calculated, in a good approximation, by the SM.

In this work we present, for the nematic Phase 5 (Merck), tests of the WEM essentially without fits of the SM parameters, and we investigate systematically the $d$ dependence of the Hopf frequency.

The threshold $\bar{V}_{c}$, the wavevector $\boldsymbol{q}_{c}$, and the Hopf frequency $\omega_{H}$ of the travelling rolls are measured, as a function of $\omega_{0}$, for three cells of different layer thickness $d$. We also measured the orientational-elastic moduli for splay $K_{11}$, twist $K_{22}$, and bend $K_{33}$, the equilibrium conductivity $\sigma_{\perp}^{\text {eq }}$, and the dielectric permittivity $\epsilon_{\perp}$ perpendicular to the director as well as their anisotropies $\left(\sigma_{a}^{\mathrm{eq}} / \sigma_{\perp}^{\mathrm{eq}}\right.$ and $\left.\epsilon_{a}\right)$. Then, together with known data for the viscosities [21], all SM parameters of Phase 5 are known apart from the small Leslie coefficient $\alpha_{1} \cdot \alpha_{1}$ and some remaining uncertainties in the equilibrium conductivity are fixed by fitting $\bar{V}_{c}$ and $\boldsymbol{q}_{c}$ within the SM. Under the assumption of a small recombination rate (as in I52), this enables a quantitative test of the dependence of $\omega_{H}$ on $\omega_{0}$. The three layer thicknesses allow a test of the predicted $d$ dependence which is independent of the material parameters. Only the overall magnitude of $\omega_{H}$ is fitted once with $\left(\mu_{\perp}^{+} \mu_{\perp}^{-}\right)^{1 / 2}$ entering as a multiplicative factor into the WEM expression. The predicted and the measured Hopf frequency agree, within the experimental errors, for all applied frequencies and all three cells, while the magnitude of $\omega_{H}$ varies by a factor of more than 50 (see Fig. 4).

In Section II, we describe the measurements, and in Section III, the relevant predictions of the WEM. Theory and experiment are compared in Section IV. Section V concludes with a discussion and points at some observed nonlinear effects which may be contained in the 
WEM.

\section{THE EXPERIMENT}

We used three cells labelled C, D, and E, respectively with areas of the rectangular plate electrodes between $10 \times 14 \mathrm{~mm}^{2}$ and $14 \times 16 \mathrm{~mm}^{2}$ defining the directions $x$ and $y$. Planar alignment along $x$ was obtained with a rubbed polyimide film which coated the transparent electrodes. The cell thickness in the $z$ direction was measured on the empty cells by an infrared spectrophotometer. Due to the large spotsize of the illuminating light beam this method yields an average thickness of $\bar{d}_{C}=24.3 \mu \mathrm{m}, \bar{d}_{D}=14.7 \mu \mathrm{m}$, and $\bar{d}_{E}=29.3 \mu \mathrm{m}$. Interference fringes indicated that the local thickness varied by $\pm 2 \mu \mathrm{m}$ around these values throughout the cell due to deformation of the plates at sealing.

The capacitance and conductance of the cells were measured by a capacitance bridge shortly after filling with the nematic mixture Phase 5 (Merck) as well as after finishing the experiments (6 weeks later). Comparing these data with the capacitance of the empty cells and neglecting stray capacitances, the dielectric permittivity $\epsilon_{\perp}$ and the electric conductivity $\sigma_{\perp}^{\text {eq }}$ at equilibrium could be obtained. These data are summarized in Table I.

The cells prepared for the measurements are too thin to determine the exact values of the anisotropies of the dielectric permittivity and the electric conductivity. Therefore we measured these parameters in a magnetic field using a thick $(>400 \mu \mathrm{m})$ cell calibrated with benzene. Table II shows the resulting data as a function of temperature.

The experimental apparatus for studying travelling waves consisted of a temperaturecontrol stage with a stability of $0.01 \mathrm{~K}$, electronics for applying the ac voltage, and a video camera with a computer-controlled imaging system. All measurements were made at (30 \pm $0.01)^{\circ} \mathrm{C}$.

The convection rolls were visualized by the shadowgraph technique [22]. The cell was illuminated by polarized light with polarization along the director, and the resulting shadowgraph signal was monitored by a video camera attached to a microscope using a 10x 
objective. The images correspond to an area of $257 \mu \mathrm{m} \times 271 \mu \mathrm{m}$ of the cell. The microscope was focused to the top of the cell to allow for a dominant linear contribution of the signal [22], where the wavelength of the shadowgraph signal coincides with that of the director distortion. Second harmonics due to the nonlinearities of the imaging technique could be seen well above threshold. The video camera was positioned to get an image of vertical rolls in the normal-roll regime where $q_{y}=0$, i.e., the rows of the pixels were parallel to the wave vector of the pattern in this regime. A few measurements were carried out in the regime of oblique rolls, so the ac frequency, where the transition between normal and oblique rolls occurs (Lifshitz point), could be estimated.

In the experimental runs, shadowgraph images were recorded for each cell at various ac frequencies in the conductive regime. The measuring program for one frequency was as follows. At first, the threshold voltage $\bar{V}_{c}$ was roughly located by an automatic iterative search. Then, a background image was recorded much below threshold. This background was subtracted on-line from all later images to remove intensity inhomogeneities due to illumination and surface irregularities. To obtain an improved value of the threshold voltage, the voltages were scanned in steps of $\Delta \epsilon \approx 0.002$ from a value above threshold $(\epsilon \approx 0.02)$ to a value below $(\epsilon \approx-0.02)$, and vice versa. Here, the control parameter $\epsilon=\bar{V}^{2} / \bar{V}_{c}^{2}-1$ denotes the deviation from the threshold. Image recording followed the change of the applied voltage with a delay of about $60-90$ seconds.

The wavenumber in the $\mathrm{x}$ direction $q_{x}$ and the frequency of the TWs at a given value of $\epsilon$ were determined by Fourier transforms of a space-time image. To obtain the space-time image, a particular row was selected in the central portion of a series of 256 images taken at consecutive time steps and this row was copied from all these images into consecutive rows of a new image yielding a 256 by 256 space-time plot. Figure 1 shows such a plot for three values of $\epsilon$. Figure 1a indicates that small-amplitude fluctuating patterns could be observed slightly below threshold. Travelling waves appear in these images as stripes tilted toward the direction of travelling. Both left and right travelling directions can be located. The horizontal periodicity in these space-time images equals to $2 \pi / q_{x}$, while the vertical one 
corresponds to the period $1 / f$ of the travelling waves.

To increase the resolution, the Fourier transformation was carried out on 2048 points with the first 256 points taken from the image (after subtracting the average intensity), and the remaining points padded with zeroes. For each row, the power spectrum $P\left(q_{x}\right)$ of the spatial frequencies (i.e., the magnitude of the squared Fourier amplitude) was calculated and averaged over all rows. The wavenumber $\bar{q}_{x}$ of the pattern was determined by a weighted average over all points in the neighbourhood of the peaks of the power spectrum corresponding to the first and second spatial harmonics,

$$
\bar{q}_{x}=\frac{\Sigma^{\prime} q_{x} \bar{P}\left(q_{x}\right)+\frac{1}{2} \Sigma^{\prime \prime} q_{x} \bar{P}\left(q_{x}\right)}{\Sigma^{\prime} \bar{P}\left(q_{x}\right)+\Sigma^{\prime \prime} \bar{P}\left(q_{x}\right)} .
$$

Here, $\bar{P}\left(q_{x}\right)$ is the average of the power spectrum over all rows attributed to the wavenumber $q_{x}, \Sigma^{\prime}$ and $\Sigma "$ sums over all points with a maximum distance of eight points from the peaks of the first and second harmonics, respectively. The Hopf frequency is determined by analogous transformations and averaging procedures for the columns.

The denominator of Eq. (11),

$$
\Sigma^{\prime} \bar{P}\left(q_{x}\right)+\Sigma^{\prime \prime} \bar{P}\left(q_{x}\right):=\bar{A}^{2},
$$

can be regarded as a measure of the averaged modulation intensity of the shadowgraph signal.

For small distortions, the modulation amplitude $\bar{A}$ is proportional to the amplitude of the director distortions [22]. The amplitude of the director distortions is expected to scale with $\epsilon^{1 / 2}$ for continuous bifurcations, at least, if subcritical fluctuations play no role. For $\epsilon$ large enough to neglect subcritical fluctuations, but small enough to remain within the linear regime of the shadowgraph technique, one has $\bar{A}^{2} \propto \epsilon$. Figure 2 shows a typical example of $\bar{A}^{2}$ vs. $\epsilon$ for cell $\mathrm{D}$ at $f_{0}=90 \mathrm{~Hz}$ corresponding to $\omega_{0} \tau_{q}=2.06$. The threshold was defined by extrapolating the first roughly linear rise of $\bar{A}^{2}$ to zero (dashed line). For $\epsilon>0.09$ one enters the nonlinear regime of the shadowgraph, which is indicated by the appearance of a second harmonic peak in the Fourier spectrum. The scatter in the data of 
Fig. 2 for $\epsilon>0.011$ is a result of the disorder appearing in the travelling pattern. There are structureless (nonperiodic) areas separating the left and right travelling waves with random space and time distribution which reduce the averaged intensity modulation accidentally.

The wavenumbers and the frequencies were calculated according to Eq.(1) as a function of $\epsilon$. The values $q_{c x}$ and $\omega_{H}$ at threshold $(\epsilon=0)$ are obtained by fitting a straight line to points in the range $-0.01<\epsilon<0.02$. To monitor the change of the conductivity during the measurements, the transition frequency $\omega_{\mathrm{cd}}$ to dielectric rolls (see Sec. IV below) was determined at the start and at the end of the experimental run for each cell. $\omega_{\mathrm{cd}}$ at the time of the measurement was determined from these values by a linear interpolation.

Despite careful preparation of the cells, there was a spatial variation of the thresholds, i.e., on increasing $\bar{V}$ from subcritical values, convection did not fill the whole cell simultaneously. To obtain the most homogeneous image possible, a local minimum or maximum of the threshold was selected in the cell for the monitored area of the measurements. In cell $\mathrm{C}$, a local minimum was chosen (convection sets in first), while in the cells D and E, a local maximum was chosen (convection sets in last; rolls appeared first at the edges of the electrode).

Elastic moduli of the substance were determined by measuring the Freedericksz thresholds in various geometries [7,23]. The magnetic field induced splay and twist Freedericksz transitions could be observed on the same planar cells used for studying the travelling waves. For magnetic and electric field induced bend transitions homeotropic cells were prepared. The field induced deformation was detected by optical methods (birefringence for splay and bend, depolarization for twist transitions). As the anisotropy of the magnetic susceptibility is unknown for Phase 5 the measurements of the threshold magnetic field yielded only a ratio of the elastic moduli, $\frac{K_{22}}{K_{11}}=0.47 ; \frac{K_{33}}{K_{11}}=1.29$. Measuring the threshold voltage in homeotropic cells we obtained $K_{33}=12.7 \times 10^{-12} N$. The error of these measurements, which are summarized in Table III, is $\pm 10 \%$. 


\section{TABLES}

TABLE I. Conductivity, dielectric permittivity, and related quantities in the cells used for the EHC experiments $\left(T=30^{\circ} \mathrm{C}\right)$.

\begin{tabular}{|c|c|c|c|c|}
\hline Quantity & Cell C & Cell D & Cell E & Comments \\
\hline Average thickness $\bar{d}[\mu \mathrm{m}]$ & 24.3 & 14.7 & 29.3 & $\begin{array}{l}\text { There is a } \pm 2 \mu \mathrm{m} \text { deviation } \\
\text { from } \bar{d} \text { throughout the cell }\end{array}$ \\
\hline Local thickness $d[\mu \mathrm{m}]$ & 23.5 & 13.4 & 27.5 & Deduced from the wavevector (see Sec. IV) \\
\hline $\begin{array}{l}\text { Conductivity } \sigma_{\perp}^{\mathrm{eq}} \\
{\left[10^{-9}(\Omega \mathrm{m})^{-1}\right]}\end{array}$ & $\begin{array}{l}8.5 \\
8.6\end{array}$ & $\begin{array}{l}16.4 \\
14.7\end{array}$ & $\begin{array}{c}7.3 \\
11.1\end{array}$ & $\begin{array}{l}\text { in fresh cell } \\
6 \text { weeks later }\end{array}$ \\
\hline Permittivity $\epsilon_{\perp}$ & $\begin{array}{l}5.34 \\
5.30\end{array}$ & $\begin{array}{l}5.38 \\
5.39\end{array}$ & $\begin{array}{l}5.24 \\
5.19\end{array}$ & $\begin{array}{l}\text { in fresh cell } \\
6 \text { weeks later }\end{array}$ \\
\hline $\begin{array}{l}\text { Range of the transition } \\
\text { frequency } \omega_{\text {cd }}[\mathrm{Hz}]\end{array}$ & $84-90$ & $138-177$ & $112-130$ & $\begin{array}{l}\text { minimum and maximum values } \\
\text { during measurements }\end{array}$ \\
\hline $\bar{\sigma}\left[10^{-9}(\Omega \mathrm{m})^{-1}\right]$ & 7.0 & 13.0 & 8.0 & $\begin{array}{l}\text { Average conductivity obtained from } \\
\text { the measured } \omega_{\mathrm{cd}} \text { (see Sec. IV) }\end{array}$ \\
\hline
\end{tabular}

TABLE II. Dielectric permittivities and electric conductivities of Phase 5 as a function of temperature for a thick cell $(400 \mu \mathrm{m})$ at $1 \mathrm{kHz}$

\begin{tabular}{cccccccc}
\hline \hline$T\left({ }^{\circ} \mathrm{C}\right)$ & $\epsilon_{\perp}$ & $\epsilon_{\|}$ & $\epsilon_{a}$ & $\sigma_{\perp}^{\mathrm{eq}}\left(10^{-9}(\Omega \mathrm{m})^{-1}\right)$ & $\sigma_{\|}^{\mathrm{eq}}\left(10^{-9}(\Omega \mathrm{m})^{-1}\right)$ & $\sigma_{a}^{\mathrm{eq}}\left(10^{-9}(\Omega \mathrm{m})^{-1}\right)$ & $\sigma_{a}^{\mathrm{eq}} / \sigma_{\perp}^{\mathrm{eq}}$ \\
\hline 20 & 5.336 & 5.100 & -0.236 & 2.89 & 4.98 & 2.09 & 0.723 \\
22 & 5.319 & 5.086 & -0.233 & 3.07 & 5.35 & 2.28 & 0.743 \\
23 & 5.305 & 5.084 & -0.221 & 3.29 & 5.65 & 2.36 & 0.717 \\
25 & 5.286 & 5.079 & -0.207 & 3.66 & 6.44 & 2.78 & 0.760 \\
27 & 5.256 & 5.058 & -0.198 & 4.25 & 7.38 & 3.13 & 0.736 \\
30 & 5.217 & 5.033 & -0.184 & 5.16 & 8.73 & 3.57 & 0.692 \\
32 & 5.185 & 5.013 & -0.172 & 5.89 & 10.17 & 4.28 & 0.727 \\
35 & 5.159 & 4.994 & -0.165 & 6.69 & 11.22 & 4.53 & 0.677 \\
\hline \hline
\end{tabular}




\section{FIGURES}

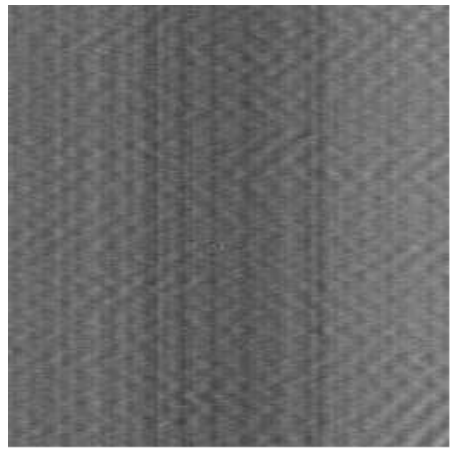

$\varepsilon=-0.001$

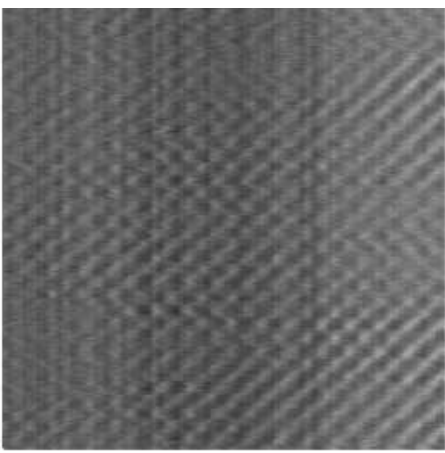

$\varepsilon=0.005$

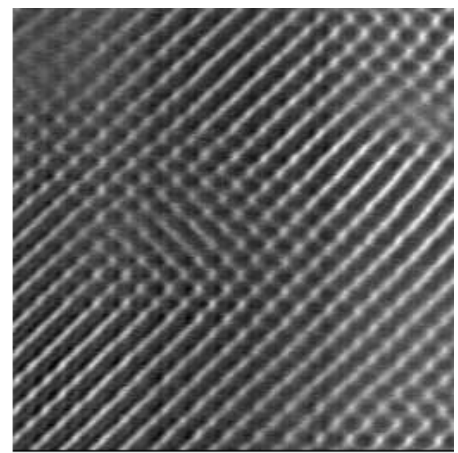

$\varepsilon=0.017$

FIG. 1. Space-time images of the travelling waves in cell $\mathrm{D}$ at $f=90 \mathrm{~Hz}$. The contrast was enhanced by a factor of 5.3 for all three images. (a) Below threshold $(\epsilon=-0.001)$. Subcritical fluctuations can be seen. (b) Sligthly above threshold $(\epsilon=0.005)$. (c) Further above threshold $(\epsilon=0.017)$. Travelling waves in both directions are observable.

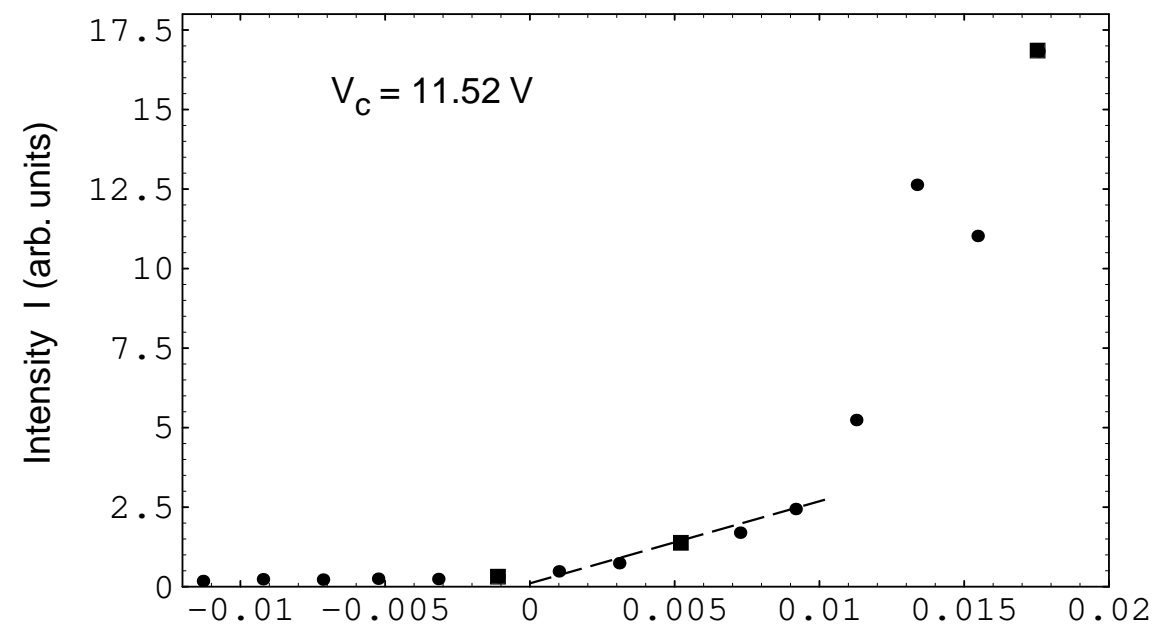

Control parameter $\varepsilon$

FIG. 2. Averaged modulation intensity $I=\bar{A}^{2}$ of the shadowgraph signal as a function of applied voltage for cell D at $f=90 \mathrm{~Hz}$. The threshold $\bar{V}_{c}$ is defined by extrapolating the first roughly linear rise of $I$ to zero (dashed line). The three squares denote the points corresponding to the space-time images in Fig. 1. 


\section{PREDICTIONS OF THE WEAK ELECTROLYTE MODEL}

In the WEM, the conductivity of the NLC is described by the drift of two species of oppositely charged freely mobile ions originating from a dissociation-recombination reaction from neutral molecules. The ionizable molecules are not specified. They can be (uncontrolled) impurities (which is assumed in most experiments on MBBA and Phase 5 and presumably accounts for the strong variations of the conductivity), or ionizable dopants (which is more controlled and realized, e.g., in I52 [17,18]).

The WEM assumes that only a small fraction of the impurities is dissociated into ionic charge carriers (weak electrolyte). This is not an essential restriction but it allows to reduce the number of material parameters not contained in the SM. For typical values for the mobilities $\left[10^{-10} \mathrm{~m}^{2} /(\mathrm{Vs})\right]$ and conductivities $\left[10^{-8}(\Omega \mathrm{m})^{-1}\right]$, the number density of both species of ions is about $3 \times 10^{20} \mathrm{~m}^{-3}$. For a molecular weight of the impurities of 100 , this corresponds to a concentration of $0.05 \mathrm{ppm}$. One has a weak electrolyte, if the number density of the impurity molecules is sufficiently higher. In the experiments employing I52 mentioned above, this is certainly fulfilled [24]. In the present work, the weak electrolyte assumption is consistent with the roughly exponential increase of the conductivities with temperature (Table II) corresponding to a dissociation energy of about $0.4 \mathrm{eV}$.

The drift velocities of the oppositely charged ions (superscript + and - ) relative to the fluid under the influence of the electric field $\boldsymbol{E}$ are given by

$$
\boldsymbol{v}_{\mathrm{drift}}^{ \pm}= \pm \underline{\underline{\mu}}^{ \pm} \boldsymbol{E}, \quad \mu_{i j}^{ \pm}=\mu_{\perp}^{ \pm}\left(\delta_{i j}+\sigma_{a}^{\prime} n_{i} n_{j}\right)
$$

defining the uniaxially anisotropic mobility tensors for the two ions. Here, $\delta_{i j}$ is the Kronecker symbol, $n_{i}$ and $n_{j}$ are components of the director, and $\sigma_{a}^{\prime}=\sigma_{a}^{\mathrm{eq}} / \sigma_{\perp}^{\mathrm{eq}}$ is the relative anisotropy of the conductivity. The local conductivity perpendicular to the director (which is constant in the $\mathrm{SM}$ ), is given in terms of the number densities $n^{ \pm}$. For charges $\pm e$, one obtains

$$
\sigma_{\perp}(\boldsymbol{r}, t)=e\left(\mu_{\perp}^{+} n^{+}+\mu_{\perp}^{-} n^{-}\right)
$$


The basic equations of the WEM are given in Ref. [20]. They are obtained by transforming the rate equations for the ionic number densities $n^{+}$and $n^{-}$into an equation for $\sigma_{\perp}(\boldsymbol{r}, t)$ describing the dynamics of the drift and of the dissociation-recombination reaction, and a conservation equation for the charge density $\rho=e\left(n^{+}+n^{-}\right)$together with the Poisson equation. The balance equations for the director components and the generalized NavierStokes equations for the fluid velocities are those of the SM.

The non-convecting basic state is nearly homogeneous, which simplifies the linear stability calculations. A one-mode Galerkin approximation for the linearized fields yields quantitative results which typically deviate by less than $3 \%$ from the fully numerical solution [24]. In this approximation, the dependence of each field on $z$ and $t$ is represented by one test function satisfying the boundary conditions and the symmetries under reflection and time translation applying in the conductive regime [20]. This one-mode approximation enables analytic formulas for the Hopf frequency, and for the threshold shift $\Delta \epsilon \equiv\left(\bar{V}_{c} / \bar{V}_{c}^{\mathrm{SM}}\right)^{2}-1$ of the WEM with respect to the SM. For details in the normal-roll regime, see Ref. [20]; the generalization to oblique rolls can be found in Ref. [17]. The threshold shift is always smaller than $1 \%$ for the experiments of this paper, so the thresholds, and also the critical wavevectors, are calculated using the SM.

For $\tilde{\omega} \tau_{d}<<\bar{V}^{2} \epsilon_{0} \epsilon_{\perp} /\left(K_{11} \pi^{2}\right)$ which is fulfilled for all measurements, the analytic expression for $\omega_{H}=2 \pi f_{H}$ is

$$
\begin{aligned}
\omega_{H} & =\tilde{\omega} \sqrt{1-\frac{1}{\left(\tau_{r e c} \tilde{\omega}\right)^{2}}}, \\
\tilde{\omega} & =\pi C^{\prime} \frac{\bar{V}_{c}^{2} \epsilon_{0} \epsilon_{\perp}}{d^{3}\left(1+\omega^{\prime 2}\right)} \sqrt{\frac{\mu_{\perp}^{+} \mu_{\perp}^{-}}{\gamma_{1} \sigma_{\perp}^{\mathrm{eq}}}} .
\end{aligned}
$$

Here, $C^{\prime}=\sqrt{\sigma_{a}^{\prime}} C$ is a dimensionless factor of order unity with $C$ given for normal rolls in Ref. 20] (see also 25]), and $\omega^{\prime}=\omega_{0} \tau_{q}\left(1+\boldsymbol{q}_{c}^{\prime 2}+\epsilon_{a}^{\prime} q_{c x}^{\prime 2}\right) /\left(1+\boldsymbol{q}_{c}^{\prime 2}+\sigma_{a}^{\prime} q_{c x}^{\prime 2}\right)$ with $\epsilon_{a}^{\prime}=\epsilon_{a} / \epsilon_{\perp}, \boldsymbol{q}=\left(q_{x}, q_{y}\right)$, and $\boldsymbol{q}^{\prime}=\boldsymbol{q} d / \pi$. In the experiments described here, one has always $0.45<C^{\prime}<0.75$ and $0.60 \omega_{0} \tau_{q}<\omega^{\prime}<0.68 \omega_{0} \tau_{q}$.

The main ingredients of Eqs. (5) and (6) can be seen by considering the two feed- 
back mechanisms resulting from the WEM. The primary instability mechanism is the CarrHelfrich mechanism of the SM. Under an applied field, an initial director bend leads via the anisotropy of the conductivity to a charge accumulation and to an electric volume force driving the fluid motion. The resulting velocity gradients couple back to the director via the rotational viscosities. The time scale of this mechanism is $\sim \tau_{d}$. The gradients of the charge accumulation, however, also excite the charge-carrier mode whose feedback tends to decrease the charge. The time scale of this second stabilizing drift mechanism is $\sim \tau_{t}^{2} / \tau_{q}$. As in binary mixtures (see, e.g., [26]), the interplay of the two mechanisms can lead to oscillations with a (Hopf) frequency proportional to the geometric mean of the two processes involved. This gives $\omega \sim \sqrt{\tau_{q} /\left(\tau_{d} \tau_{t}^{2}\right)}$, which is Eq. (6), up to a dimensionless prefactor.

The drift mechanism is only effective if the charge-carrier mode can build up sufficiently, i.e., for a small recombination rate. This leads to the square root in Eq. (5) and to the condition $\tau_{\text {rec }}^{-1}<\tilde{\omega}$ for the occurrence of a Hopf bifurcation to TWs. The condition $\tilde{\omega}=\tau_{\text {rec }}^{-1}$ gives the codimension-two point separating the regime of stationary rolls $\left(\tau_{\text {rec }}^{-1}>\tilde{\omega}\right)$ from that of TWs. This condition scales with $d^{-3},\left(\sigma_{\perp}^{\mathrm{eq}}\right)^{-1 / 2}$, and $\bar{V}_{c}^{2} /\left(1+\omega^{\prime 2}\right)$, hence a Hopf bifurcation is favoured by thin cells, clean cells (low $\sigma_{\perp}^{\mathrm{eq}}$ ), and by high values of the applied frequencies in case of materials with $\epsilon_{a}<0$ (for $\epsilon_{a}<0$, the factor $\bar{V}_{c}^{2} /\left(1+\omega^{\prime 2}\right.$ ) increases with $\omega_{0}$ and contains the main part of the dependence on $\left.\omega_{0}\right)$.

In contrast to former experiments on Phase 5 [2, 19], we observed TWs for all applied frequencies in the three cells. This is presumably due to a smaller concentration of impurities. The Hopf condition is most restrictive for the thickest cell at the lowest frequencies. The experimental points for this cell (squares in Fig. 3a) suggest that one is still far away from the codimension-two point. With Eq. (5), this means that $\omega_{H} \approx \tilde{\omega}$, i.e., $1 / \tau_{\text {rec }}$, can be set to zero in the calculations.

In this case, the WEM predictions contain $\left(\mu_{\perp}^{+} \mu_{\perp}^{-}\right)^{1 / 2}$ as the only non-SM parameter, which appears in $\omega_{H} \approx \tilde{\omega}$, as a simple multiplicative factor. The WEM predicts that $\tilde{\omega}$ is proportional to $d^{-3}\left(\sigma_{\perp}^{\mathrm{eq}}\right)^{-1 / 2}$ and increases with the ac frequency. In the following section, these predictions will be compared with the experiment. 


\section{COMPARISON BETWEEN EXPERIMENT AND THEORY}

The WEM gives $\bar{V}_{c}, \boldsymbol{q}_{c}$, and the Hopf frequency $\omega_{H}$, as a function of the applied frequency $\omega_{0} \tau_{q}$ normalized to the inverse of the charge relaxation time $\tau_{q}$. The values for $\bar{V}_{c}$ and $\boldsymbol{q}_{c}$ differ by less than $1 \%$ from those of the SM. In a good approximation, $\bar{V}_{c}\left(\omega_{0} \tau_{q}\right)$ and $q_{c}\left(\omega_{0} \tau_{q}\right) d / \pi$ do not depend on $d$ or on $\sigma_{\perp}^{\text {eq }}[3]$, the only parameters that are significantly different in the three cells. All experimental points for $\bar{V}_{c}$ and $q_{c}$ should lie on one curve, if one plots $\bar{V}_{c}$ and $q_{c} d / \pi$ against $\omega_{0} \tau_{q}$. Similarly, the WEM requires that the experimental points for $\omega_{H} d^{3}\left(\sigma_{\perp}^{\mathrm{eq}}\right)^{1 / 2}$ should lie on one line. To verify this, requires knowledge of $\tau_{q} \propto 1 / \sigma_{\perp}^{\text {eq }}$ for each experimental point.

The conductivity turned out to vary somewhat irregularly during the experimental runs (Table I). The relative change of the conductivities during the measurements can be assessed by determining, for each measurement of the set $\bar{V}_{c}, q_{c}$, and $\omega_{H}$, the transition frequency $\omega_{\mathrm{cd}}$ where the rolls of the conductive regime cross over to dielectric rolls [1], which can be easily identified. This frequency is related to the "cutoff frequency" $\omega_{\text {cutoff }}$ where the threshold of the conductive regime diverges in the one-mode approximation. The ratio $\omega_{\mathrm{cd}} / \omega_{\text {cutoff }}$ depends on $d$, but is constant for one cell $\left(\omega_{\text {cutoff }} / \omega_{\text {cd }}=0.68 \cdots 0.74\right.$ for the three cells $)$. Since the cutoff frequency in the SM is proportional to $\sigma_{\perp}^{\text {eq }}\left(\omega_{\text {cutoff }}=5.57 \tau_{q}^{-1}\right.$ for the parameters of Table III), the actual conductivity can be written as

$$
\sigma_{\perp}^{\mathrm{eq}}=\frac{\omega_{\mathrm{cd}}}{\bar{\omega}_{\mathrm{cd}}} \bar{\sigma},
$$

where $\bar{\omega}_{\text {cd }}$ is defined as the average of the transition frequencies over all measurements in one cell (C,D, or E) and the "average conductivity" $\bar{\sigma}$ must be determined separately.

Figure 3a shows the resulting threshold voltages (from the SM) as a function of $\omega_{0} \tau_{q}=$ $\omega_{0} \epsilon_{0} \epsilon_{\perp} / \sigma_{\perp}^{\text {eq }}$ with $\sigma_{\perp}^{\text {eq }}$ from Eq. (1) (continuous curve) together with the experimental results. For each of the cells, $\bar{\sigma}$ was fitted to the threshold curves of the SM with the other parameters taken from Table III in Appendix A. Of course, the resulting value of $\bar{\sigma}$ must be consistent with the independent conductivity measurements (Table I) taken some time before and after 
the measurements of the patterns. Table I shows that $\bar{\sigma}$ deviates by less than $25 \%$ from these measurements, which is within the variations of the conductivities as estimated from the variations of $\omega_{\mathrm{cd}}$. The unknown viscosity $\alpha_{1}$ was adjusted as well. In the normal-roll regime (all but the measurements corresponding to the three leftmost triangles are in this regime), the influence of $\alpha_{1}$ on the threshold and on $\omega_{H}$ is negligible. In contrast, the tendency to oblique rolls is increased by increasing $\alpha_{1}$; so $\alpha_{1}$ was fitted to give the correct frequency for the transition from normal to oblique rolls, the Lifshitz point (in the SM).

Figure $3 \mathrm{~b}$ shows the normalized $x$-component $q_{c x} d / \pi$ of the wavevector. Taking for $d$ the average thicknesses measured on the empty cells $(\bar{d}$, Sec. II) gives a systematic deviation $(5 \%-10 \%)$ of the experimental points from the curves from the SM. One can attribute this to the fact that the actual thickness at the position of the measurement may differ from $\bar{d}$ (see Sec. II). The systematic deviations can mostly be removed by using slightly reduced thickness values $d_{C}=23.5 \mu \mathrm{m}, d_{D}=13.4 \mu \mathrm{m}$, and $d_{E}=27.5 \mu \mathrm{m}$ (Table I). This correction (a reduction by $0.8 \mu \mathrm{m}$ for cell $\mathrm{C}$, by $1.4 \mu \mathrm{m}$ for cell $\mathrm{D}$, and $1.8 \mu \mathrm{m}$ for cell $\mathrm{E}$ ) is within the limits of deviation indicated by the interference fringes $( \pm 2 \mu \mathrm{m})$. Hence for the plots presented in this paper we assumed these reduced thickness values. The agreement for these thicknesses is reasonable, although the theoretical frequency dependence (from the SM) is somewhat too steep at larger $\omega_{0} \tau_{q}$. The kink in the theoretical curve at $\omega_{0} \tau_{q}=0.8$ is due to the Lifshitz point.

In Figure 4, we show the main result of this paper, namely the measured Hopf frequencies together with the WEM predictions, Eq. (5), under the assumption of a long recombination time where $\omega_{H}=\tilde{\omega}$. The overall magnitude of $\omega_{H}$ fixes the mobilities to

$$
\sqrt{\mu_{\perp}^{+} \mu_{\perp}^{-}}=1.1 \times 10^{-10} \mathrm{~m}^{2} /(\mathrm{Vs})
$$

but nothing else can be fitted. Since in the normal-roll regime $\alpha_{1}$ plays a minor role for the dependence on $\omega_{0}$, the $d$ dependence and the dependence on $\omega_{0}$ provided by the theory are in effect free from unknown or adjustable parameters.

The measurements for the thickest cell (squares) and the thinnest cell (triangles) agree 
with the WEM curve nearly everywhere within the experimental error of about 25\%; the Hopf frequency of cell $\mathrm{C}$ (circles) is systematically higher $(\approx 25 \%)$ than the predicted one. This has to be compared with the wide range covered by the actual Hopf frequencies. In the experiments, the Hopf frequency varies from about $0.4 \mathrm{~s}^{-1}$ (cell $\mathrm{E}$ at $\omega_{0} \tau_{q}=0.8$ ) to more than $22 \mathrm{~s}^{-1}$ (cell D at $\omega_{0} \tau_{q}=4.0$ ), i.e., by a factor of more than 50. For a given external frequency, the Hopf frequency of cell D is about seven times that of cell E. 

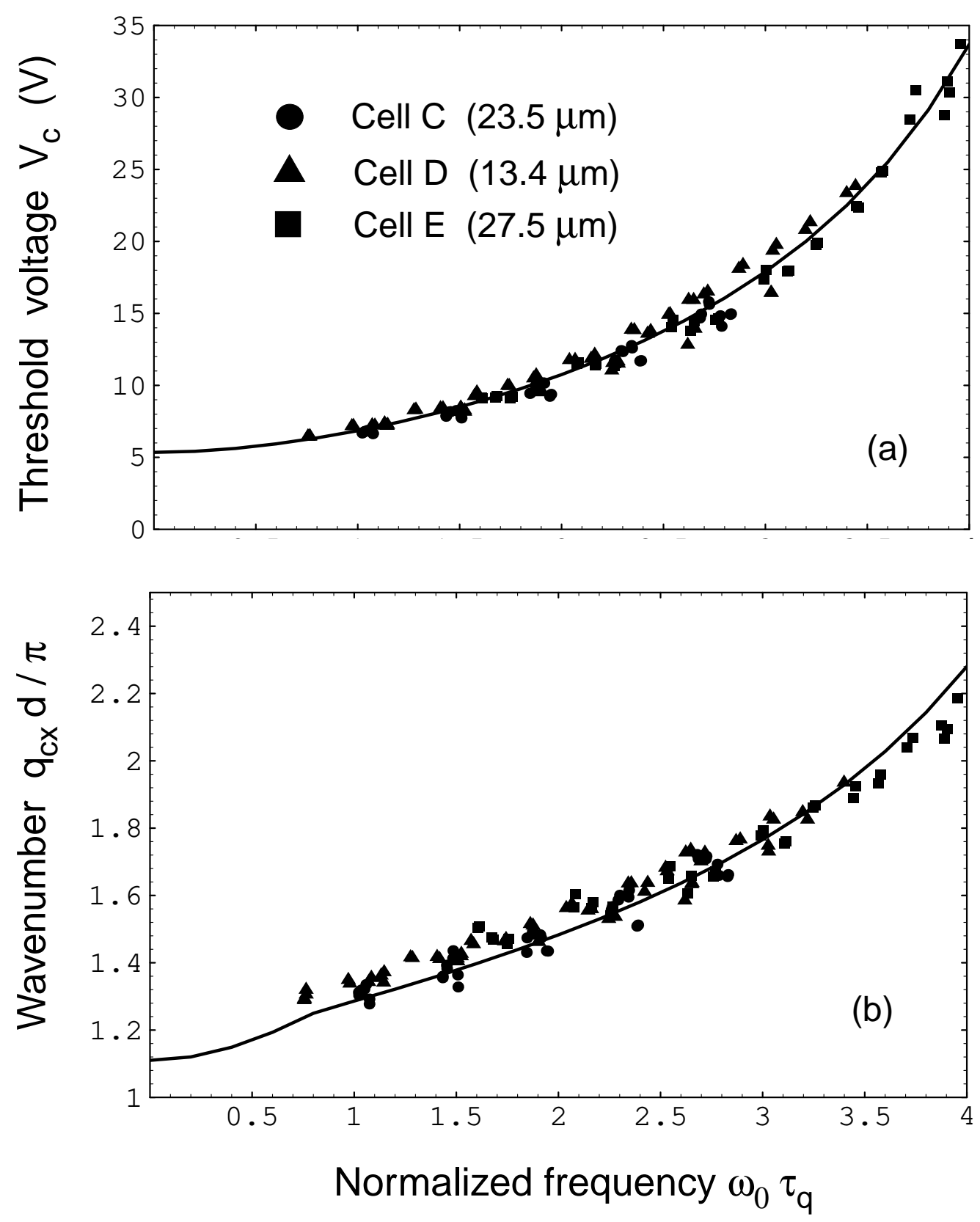

FIG. 3. (a) Threshold voltage, and (b) wavenumber $q_{c x}$ as a function of $\omega_{0} \tau_{q}$. The conductivity $\sigma_{\perp}^{\text {eq }}$ in $\tau_{q}$ is calculated individually for each point from $\omega_{\text {cd }}$, see the main text. The experimental points of the three cells (circles, squares, and triangles) should lie on one curve for $\bar{V}_{c}$ and $q_{c x} d / \pi$, respectively. The curves are the SM predictions for the material parameters of Phase 5 given in Appendix A with the viscosity $\alpha_{1}$ fitted to $-0.35 \gamma_{1}$. The WEM predictions for $\bar{V}_{c}$ and $q_{c x}$ cannot be distinguished from that of the SM on this scale. 


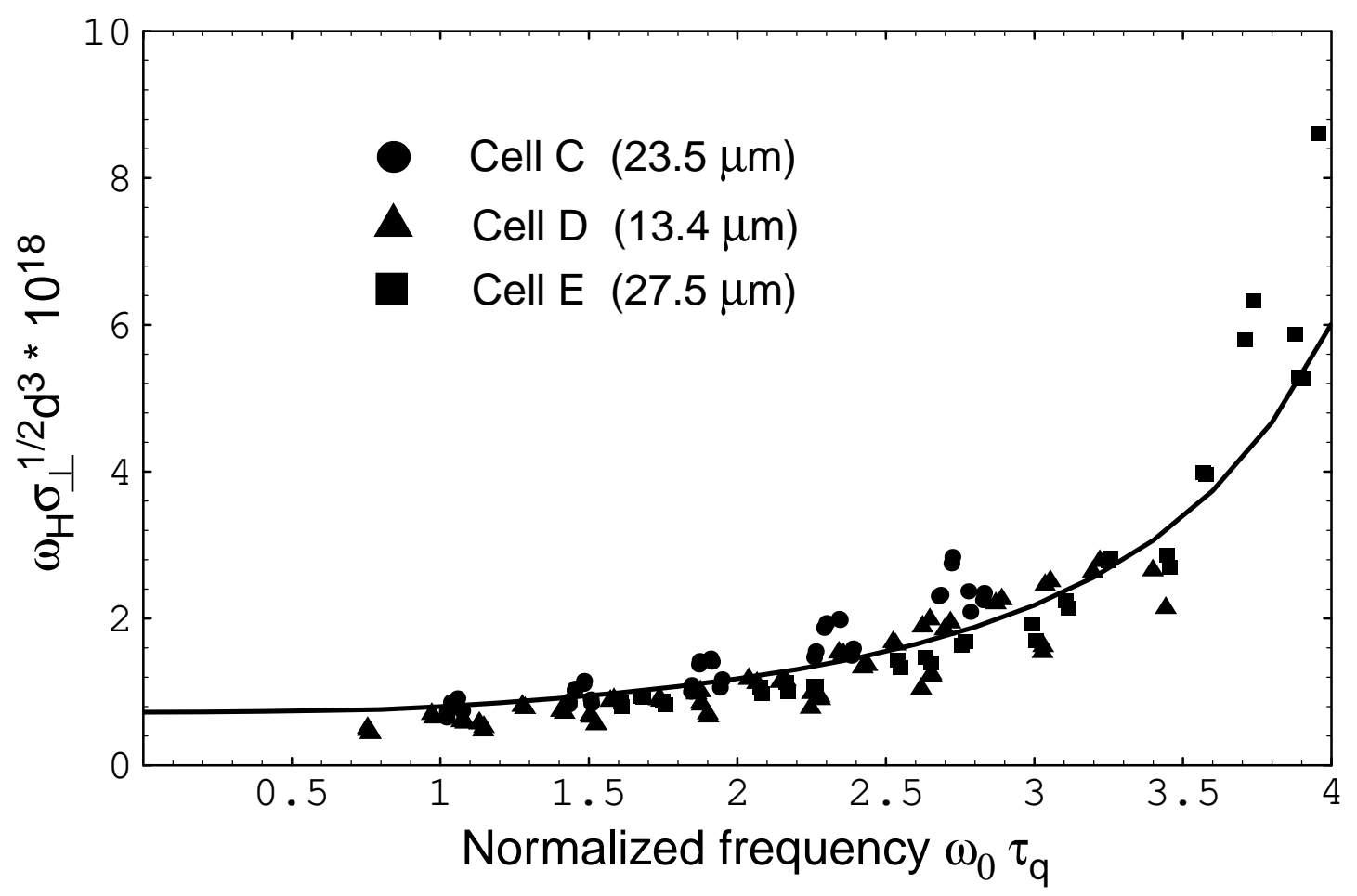

FIG. 4. The Hopf frequency of the travelling rolls multiplied by $\left(\sigma_{\perp}^{\text {eq }}\right)^{1 / 2} d^{3}$, is shown as a function of the normalized applied frequency $\omega_{0} \tau_{q}$. The circles, squares, and triangles denote the experimental results for the three cells. The curve is the universal WEM prediction for the material parameters of Phase 5 (Appendix A) and $\sqrt{\mu_{\perp}^{+} \mu_{\perp}^{-}}=1.1 \times 10^{-10} \mathrm{~m}^{2} /(\mathrm{Vs})$. For $\omega_{0} \tau_{q}=0$, this curve corresponds to $\omega_{H}=0.67 \mathrm{~s}^{-1}, 2.65 \mathrm{~s}^{-1}$, and $0.37 \mathrm{~s}^{-1}$, for the cells $\mathrm{C}, \mathrm{D}$, and E, respectively.

\section{CONCLUSION}

Our results represent the most stringent test for the WEM model so far. The frequency dependence of the threshold $\bar{V}_{c}$ and, to less extent, the critical wavevector $\boldsymbol{q}_{c}$, involving in essence only SM quantities, has been tested previously fairly well in the conductive range [1.9]. Also $\alpha_{1}$ was fitted to obtain the correct Lifshitz point, but this is of little influence in the normal-roll range considered here mainly. The Hopf frequency $\omega_{H}$ predicted by the WEM deviates from the measured ones by at most $\approx 25 \%$ in a range of parameters where $\omega_{H}$ varies by a factor of about 50 . This was achieved by fitting only the parameter $\sqrt{\mu_{\perp}^{+} \mu_{\perp}^{-}}$ appearing as an overall factor. 
The value of $\sqrt{\mu_{\perp}^{+} \mu_{\perp}^{-}}$given in Eq.(8) compares reasonably well with a number of measurements of the quantity $\mu=\mu_{\perp}^{+}+\mu_{\perp}^{-}$in different materials, namely (in units of $10^{-10} \mathrm{~m}^{2} /(\mathrm{Vs})$ ) $\mu=0.18$ [28], 1 [10], 3.71 [30], $1 . .10$ [31], 10 [27]. Little appears to be known about the ratio $\mu_{\perp}^{+} / \mu_{\perp}^{-}$.

Our results indicate that the recombination time $\tau_{\text {rec }}$ is well above $1 / \omega_{H}$ even for the smallest Hopf frequency measured, which means $\tau_{\text {rec }}$ is larger than about 5s. Such long times may seem surprizing, however, literature values for MBBA range from $10^{-3} \mathrm{~s}$ [27] to $2.7 \times 10^{4} \mathrm{~s} 28$. Thus in our experiments one is well away from the codimension-2 point that separates the stationary regime from the Hopf regime. Let us point out that a weakly nonlinear analysis predicts the bifurcation to be typically subcritical near the codimension- 2 point on the stationary side [24]. This appears to explain the slight hysteresis observed in some high-sensitivity experiments [17,32,16]. The fact that a hysteresis is sometimes also observed on the Hopf bifurcation side [32,16] is not disturbing because there is evidence that this is due to higher-order effects in the amplitude [34]. The theoretical analysis is consistent with this fact.

For further testing of the WEM an independent determination of both $\tau_{\text {rec }}$ and the mobilities is desirable. This could be done by measuring the transient current response to suitable voltage signals in cells with well defined boundary conditions (e.g., blocking electrodes) $33,29,28$.

\section{ACKNOWLEDGMENTS}

We wish to thank J. Peinke and M. Scheuring for help with the experiments and with the characterization of the material. R. Stannarius made us aware of Ref. [21] where the viscosities of Phase 5 were measured. A.B. and N.É. appreciate the support and kind hospitality of the University of Bayreuth. The nematic Phase 5 was kindly made available for the measurements by Merck, Darmstadt. This work was financially supported by the Volkswagen-Stiftung, the Hungarian Research Grant No. OTKA T014957 and the EU TMR 
project ERB FMRX-CT 96-0085. 


\section{APPENDIX A: MATERIAL PARAMETERS FOR PHASE 5 AT $30{ }^{\circ} \mathrm{C}$}

The parameters $K_{11}, K_{22}, K_{33}, \epsilon_{\perp}, \epsilon_{a}, \sigma_{\perp}^{\text {eq }}$, and $\sigma_{a}^{\text {eq }}$ were measured in this work. The actual value of $\sigma_{\perp}^{\mathrm{eq}}$ varied considerably and was determined individually for each measurement by Eq. (7) with $\bar{\sigma}$ from Table I. There are published data for the dielectric anisotropy 35 which differ somewhat, but these data seem to be rather inprecise. The Leslie viscosities $\alpha_{2}$ to $\alpha_{6}$ were determined from the viscosities $\eta_{1}, \eta_{2}, \eta_{3}$, and $\gamma_{1}$ of Ref. [21] by the relations $\alpha_{2}=\left(\eta_{2}-\eta_{1}-\gamma_{1}\right) / 2, \alpha_{3}=\left(\eta_{2}+\eta_{1}-\gamma_{1}\right) / 2, \alpha_{4}=2 \eta_{3}, \alpha_{6}=\left(\eta_{1}+3 \eta_{2}-4 \eta_{3}-\gamma_{1}\right) / 2$, and the Onsager relation $\alpha_{5}=\alpha_{6}-\alpha_{2}-\alpha_{3}$.

TABLE III. Material parameters for Phase 5

\begin{tabular}{|c|c|c|c|}
\hline Parameter & Unit & Value at $30^{\circ} \mathrm{C}$ & Source and comments \\
\hline$K_{11}$ & $10^{-12} \mathrm{~N}$ & 9.8 & Sec. II \\
\hline$K_{22}$ & $"$ & 4.6 & $"$ \\
\hline$K_{33}$ & $"$ & 12.7 & $"$ \\
\hline$\sigma_{a} / \sigma_{\perp}^{\mathrm{eq}}$ & - & 0.7 & Table II \\
\hline$\epsilon_{\perp}$ & - & $5.19 \cdots 5.39$ & Tables I, II \\
\hline$\epsilon_{a}$ & - & -0.184 & Table II; see also Ref. 35 \\
\hline$\alpha_{1}$ & $10^{-3} \mathrm{Ns} / \mathrm{m}^{2}$ & -39 & Fit to the Lifshitz point \\
\hline$\alpha_{2}$ & $"$ & -109.3 & Kneppe, et al, Ref. 21] \\
\hline$\alpha_{3}$ & $"$ & 1.5 & $"$ \\
\hline$\alpha_{4}$ & $"$ & 56.3 & $"$ \\
\hline$\alpha_{5}$ & $"$ & 82.9 & $"$ \\
\hline$\alpha_{6}$ & $"$ & -24.9 & $"$ \\
\hline$\sqrt{\mu_{\perp}^{+} \mu_{\perp}^{-}}$ & $10^{-10} \mathrm{~m}^{2} /(\mathrm{Vs})$ & 1.1 & Fit to $\omega_{H}$ \\
\hline$\tau_{\text {rec }}^{-1}$ & $\mathrm{~s}^{-1}$ & $<0.2$ & Hopf bifurcation in all cells \\
\hline
\end{tabular}




\section{REFERENCES}

[1] L. Kramer and W. Pesch, Annu. Rev. Fluid Mech. 27, 515 (1995); L. Kramer and W. Pesch, Electrohydrodynamic instabilities in nematic liquid crystals, in L. Kramer and A. Buka, editors, Pattern Formation in Liquid Crystals, Springer, (NY 1996).

[2] I. Rehberg, B. L. Winkler, M. de la Torre Juarez, S. Rasenat, and W. Schöpf, Advances in Solid State Physics 29, 35 (1989).

[3] E. Bodenschatz, W. Zimmermann, and L. Kramer, J. Phys. France 49, 1875 (1988).

[4] W. Helfrich, J. Chem. Phys. 51, 4092-4105 (1969).

[5] J. L. Ericksen, Arch. Ration. Mech. Anal. 4, 231 (1960).

[6] F. M. Leslie, Quart. J. Mech. Appl. Math. 19, 357 (1966).

[7] P. de Gennes and J. Prost, The Physics of Liquid Crystals, Clarendon, (Oxford 1993).

[8] W. Zimmermann and L. Kramer, Phys. Rev. Lett. 55, 402-5 (1985).

[9] L. Kramer, E. Bodenschatz, W. Pesch, W. Thom, and W. Zimmermann, Liquid Crystals 5(2), 699-715 (1989).

[10] L. M. Blinov, Electrooptical and Magnetooptical Properties of Liquid Crystals, John Wiley, (NY 1983).

[11] M. Kaiser and W. Pesch, Phys. Rev. E 48, 4510-28 (1993).

[12] W. Decker and W. Pesch, J.Phys.II France 4, 493 (1994).

[13] W. Decker, Phd dissertation, University of Bayreuth, (1995).

[14] S. Kai and K. Hirakawa, Prog. Theor. Phys. Suppl. 64, 212 (1978).

[15] I. Rehberg, S. Rasenat, and V. Steinberg, Phys. Rev. Lett. 62, 756-59 (1989).

[16] I. Rehberg, S. Rasenat, M. de la Torre Juarez, W. Schöpf, F. Hörner, G. Ahlers, and 
H. R. Brand, Phys. Rev. Lett 67, 596 (1991).

[17] M. Dennin, M. Treiber, L. Kramer, G. Ahlers, and D. Cannell, Phys. Rev. Lett. 76, 319 (1996).

[18] M. Dennin, D. S. Cannell, and G. Ahlers, Mol. Cryst. Liq. Cryst 261, 377 (1995).

[19] A. Joets and R. Ribotta, Phys. Rev. Lett. 60, 2164 (1988).

[20] M. Treiber and L. Kramer, Mol. Cryst. Liq. Cryst 261, 311 (1995).

[21] H. H. Graf, H. Kneppe, and F. Schneider, Molecular Physics 77, 521 (1992).

[22] S. Rasenat, G. Hartung, B. Winkler, and I. Rehberg, Exp. in Fluids 7, 412 (1989).

[23] H. J. Deuling, Solid State Phys. Suppl. 14, 77 (1978).

[24] M. Treiber, On the Theory of the Electrohydrodynamic Instability in Nematic Liquid Crystals near Onset, Phd dissertation, University of Bayreuth, (1996).

[25] There is a printing error in Eq. (38) of Ref. [20]. The factor $\left(1-\frac{\epsilon_{a}}{\epsilon_{q}} L_{n n} q^{2}\right)$ should be replaced by $\left(L_{n n}-\frac{\epsilon_{a}}{\epsilon_{q}} q^{2}\right)$.

[26] M. C. Cross and P. C. Hohenberg, Rev. Mod. Phys. 65(3), 851 (1993).

[27] R. Turnbull, J. Phys. D6, 1745 (1973).

[28] G. Brière, R. Herino, and F. Mondon, Mol. Cryst. Liq. Cryst. 19, 157 (1972).

[29] A. Sugimura, et al., Phys. Rev. B 43, 8272 (1991).

[30] R. Chang and J. Richardson, Mol. Cryst. Liq. Cryst 28, 189 (1973).

[31] P.G. de Gennes, Comments on Solid State Physics 3, number 5, page 148 (1971).

[32] I. Rehberg, F. Hörner, L.Chiran, H. Richter, and B. Winkler, Phys. Rev. A 44, 7885 (1991).

[33] H. Naito, M. Okuda, and A. Sugimura, Phys. Rev. A 44, 3434 (1991). 
[34] F. Hörner, private communication.

[35] Data sheets "N5 Licristal" from Merck, Darmstadt, Germany (1982). 